\title{
Gate-Tunable Photoemission from Graphene Transistors
}

\author{
Mehmet Copuroglu, ${ }^{\dagger}$ Pinar Aydogan, ${ }^{\dagger}$ Emre O. Polat, ${ }^{\dagger}$ Coskun Kocabas, ${ }^{*}{ }^{\ddagger}$ and Sefik Süzer* ${ }^{\dagger}$ \\ ${ }^{\dagger}$ Department of Chemistry and ${ }^{\ddagger}$ Department of Physics, Bilkent University, Ankara 06800, Turkey
}

ABSTRACT: In this Letter, we report gate-tunable X-ray photoelectron emission from back-gated graphene transistors. The back-gated transistor geometry allows us to study photoemission from graphene layer and the dielectric substrate at various gate voltages. Application of gate voltage electrostatically dopes graphene and shifts the binding energy of photoelectrons in various ways depending on the origin and the generation mechanism(s) of the emitted electrons. The gate-induced shift of the Fermi energy of graphene alters the binding energy of the $\mathrm{C}$ 1s electrons, whereas the electric field of the gate electrodes shift the binding energy of core electrons emitted from the gate dielectric underneath the graphene layer.
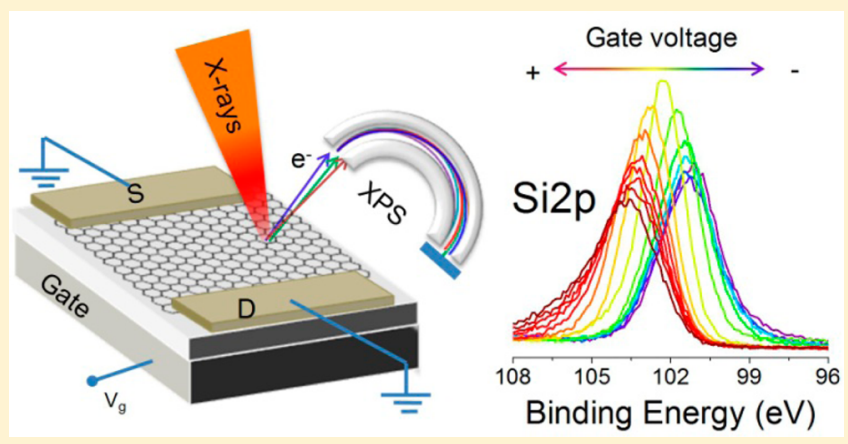

The gradual change of the local potential through depths of the gate dielectric provides quantitative electrical information about buried interfaces. Our results suggest that gate-tunable photoemission spectra with chemically specific information linked with local electrical properties opens new routes to elucidating operation of devices based especially on layered materials.

KEYWORDS: XPS, graphene transistor, gate tunable, binding energy shifts, gate dielectric, $2 \mathrm{D}$ crystals
$G$ raphene, the two-dimensional (2D) crystal of carbon, has unique electrically tunable properties. ${ }^{1-3}$ Owing to its monatomic thickness and delocalized p-orbitals, graphene forms $2 \mathrm{D}$ electron/hole gas with gate-tunable charge density. Other tunable 2D electronic systems, ${ }^{4}$ such as Si-inversion layer or GaAs heterostructures, are usually buried under a metal gate electrode. Thick metal gate-electrodes and shallow penetration depth of electron-based surface tools have been the obstacles for detailed chemical characterization of the buried 2D electronic systems. Back-gated graphene transistors provide unique device geometry for tunable electronic systems that can be probed by surface characterization tools under voltage bias. In this Letter, we report on gate tunable X-ray photoelectron emission from a graphene field effect transistor.

Many features of graphene can be tuned by electrostatic doping. For example, optical absorption, plasma frequency, Raman cross-section and electron-phonon coupling in graphene can be tuned by electrostatic gating. ${ }^{1,3,5}$ The Fermi energy of graphene varies with charge density as $E_{\mathrm{f}}=$ $\hbar \nu_{\mathrm{F}}(\pi n)^{1 / 2}$ where $\nu_{\mathrm{F}}$ is the Fermi velocity and $n$ is the areal charge density. Using back-gated transistors, Fermi energy of $300 \mathrm{meV}$ with a charge density up to $5 \times 10^{12} 1 / \mathrm{cm}^{2}$ can be achieved. ${ }^{6}$ The variation of Fermi energy results in blocking of interband electronic transitions, an increase in the plasma frequency and interference of possible electronic pathways. Raman spectroscopy, optical reflection spectroscopy, and scanning probe microscopy of graphene transistors have been extensively used to investigate the tunable nature of graphene. $5,7-18$

These optical spectroscopy techniques, however, can only provide indirect information about the variations in the charge density and Fermi energy without chemical specificity. For example, scanning Kelvin-probe microscopy is a powerful method for probing such variations but is not capable of yielding chemical information. ${ }^{19,20}$ Photoelectron emissionbased techniques such as UPS (ultraviolet photoelectron spectroscopy) are alternative techniques and have been extensively used to detect and quantify changes in the workfunction as well as in the Dirac-Point of graphene-based materials and devices. ${ }^{21-26}$ XPS (X-ray photoelectron spectroscopy) is even more attractive because it can give local voltage variations in a chemically resolved fashion derived from the binding energy shifts of the corresponding core-levels. Use of XPS for chemical identification of the species formed after certain chemical and/or electrochemical modification of graphene or carbon based materials is common. ${ }^{27-41}$

One particular aspect of XPS technique is its sensitivity to the potentials developed as a result of a particular chemical/ physical process, best exemplified by a recent work of Tarabek et al., where they reported that ex situ spectra of the $C 1$ s and the $\mathrm{C}_{\mathrm{KLL}}$ Auger peaks of the carbon-nanotube samples shifted 0.5 and $-0.5 \mathrm{eV}$ when the samples were previously doped at electrode potentials of -1.5 (n-doping) and $1.5 \mathrm{~V}$ (p-doping), respectively. ${ }^{42}$ Another important aspect of XPS is its ability to detect the shifts in the binding energy position of the peaks as a result of imposed external voltage stresses in the form of direct current $(\mathrm{dc})$ or alternating current $(\mathrm{ac})$ stimuli. This variation has been extensively used by our group to probe electrical properties of the materials' surfaces. ${ }^{43,44}$ In our previous work, we imposed a $6 \mathrm{~V}$ dc voltage bias across a graphene sheet of $2 \times$

Received: March 5, 2014

Revised: March 26, 2014

Published: March 31, 2014 

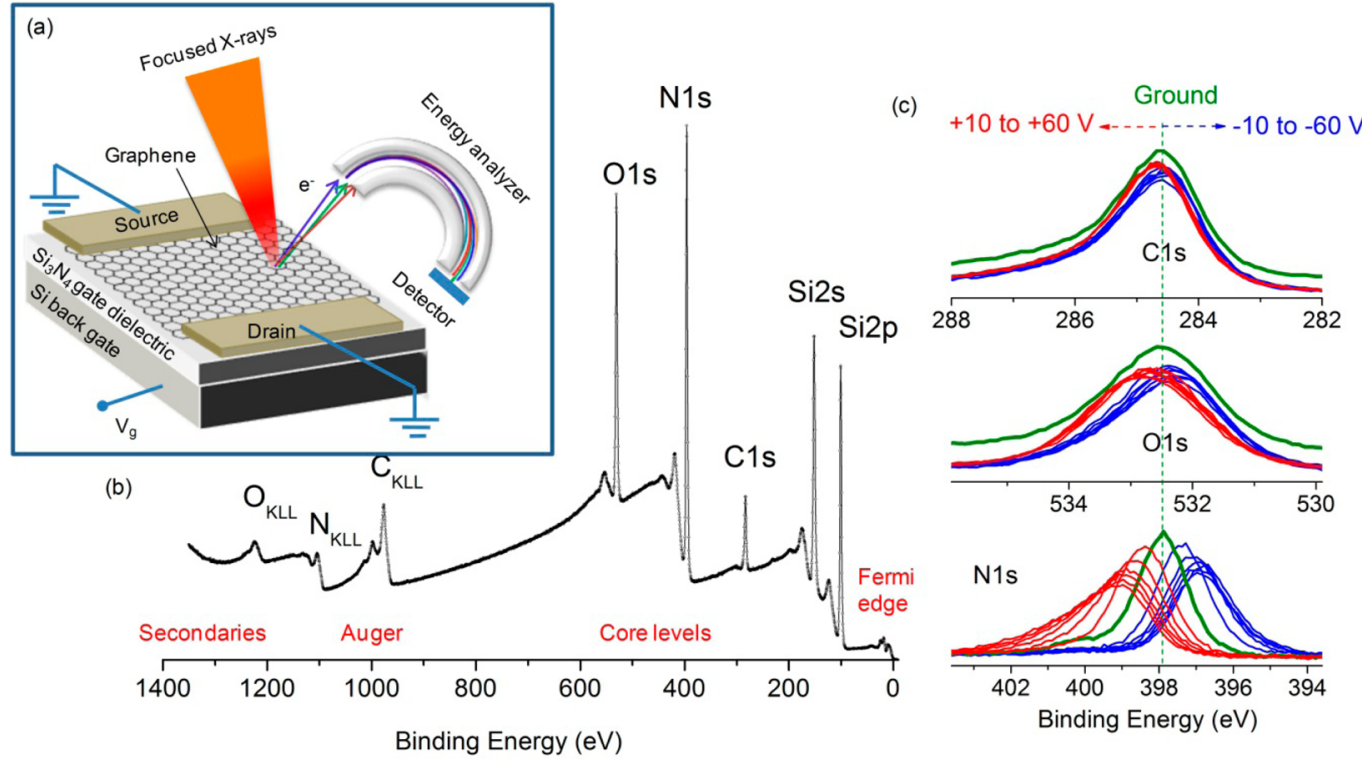

Figure 1. (a) Schematic representation of the back-gated graphene field effect transistor and the experimental setup used to measure the gate-tunable XPS spectra. (b) Full range of XPS spectra from the transistor recorded while the graphene layer and gate electrodes are grounded. (c) C 1s, O 1s, and $\mathrm{N} 1 \mathrm{~s}$ regions recorded while the gate is biased from -60 to $60 \mathrm{~V}$. Note that the $x$-scale is different for the three regions, in the right inset.

$3 \mathrm{~mm}$ between the two gold electrodes and measured the voltage variations throughout the entire sheet by monitoring the binding energy position of the $\mathrm{C} 1 \mathrm{~s}$ peak, which varied smoothly from 290.75 to $284.75 \mathrm{eV}$, exhibiting position dependent shift of 6.0 down to $0.0 \mathrm{~V} .^{44}$ On the other hand, such variations were not very smooth, and revealed the presence of cracks, after exposing the graphene sheet to mild oxidizing oxygen plasma. Hence, we claimed that the measured 3 -fold increase in the resistance of the graphene sheet (from 0.4 in its pristine to $1.2 \mathrm{k} \Omega$ in its oxidized form) was not only due to the introduction of oxidized carbon moieties but also to the presence of various defects and/or cracks created by the plasma. Here we developed a new method to study gatetunable photoemission by applying a gate voltage that induces free carriers on graphene and shifts the Fermi energy. This unique experimental step allows us investigate the gatetunability of the XPS peaks and discuss possible mechanism(s) causing the observed findings.

The present work differs significantly from our previous one in two respects. (i) The device structures are different, because in the latter the graphene layer was deposited on an insulator (quartz), which performed as a resistor and electrostatic gating was not possible. In the present work, we probed graphene in a transistor geometry, and graphene acts as a semiconductor. (ii) The physical mechanisms behind the observed binding energy shifts are different, because the binding energy shift of $C 1 \mathrm{~s}$ in the previous work were due to the local voltage imposed by large current (in the range of $\mathrm{mA}$ ) passing from one side of the graphene sheet to the other (IR drop), whereas in the present case, the binding energy of $\mathrm{C} 1 \mathrm{~s}$ of graphene is due to the shift in the Fermi energy and very little or no significant current is encountered. However, both methodologies are unique, because they both probe different electrical properties of devices under operation, and both have never been reported before.

The left-inset (Figure 1a) shows the schematic drawing of a back-gated graphene transistor and X-ray photoelectron spectrometer. We used a Thermo Fisher K-alpha electron spectrometer with a monochromatic $\mathrm{Al} \mathrm{K} \alpha$ X-ray source $(h \nu=$ $1486.6 \mathrm{eV}$ ) and modified it in order to apply external voltage bias to the samples. The samples consisted of large area $(1 \mathrm{~cm}$ $\times 1 \mathrm{~cm}$ ) graphene transfer-printed on Si substrates coated with $100 \mathrm{~nm} \mathrm{Si}{ }_{3} \mathrm{~N}_{4}$. The graphene samples were grown on $25 \mu \mathrm{m}$ thick copper foils using chemical vapor deposition, then transfer printed on $\mathrm{Si}_{3} \mathrm{~N}_{4}$ coated $\mathrm{Si}$ wafers. ${ }^{45} \mathrm{We}$ used conductive carbon tapes (Ted Pella Inc.) to contact the graphene layer. The distance between the source/drain electrodes is $1 \mathrm{~cm}$. Figure $1 \mathrm{~b}$ shows the recorded X-ray photoelectron spectra from the graphene field effect transistor, when the source/drain electrodes are grounded. The content of the spectra is very rich. Gate-dependence of the XPS spectra, namely, C 1s (graphene), Si 2p (gate dielectric), N 1s (gate dielectric), and $\mathrm{O} 1 \mathrm{~s}$ (surface oxide of the gate dielectric) regions under different gate voltages shows different behavior depending on the origin of the emitted photoelectrons. Behavior of the Si $2 p$ (gate dielectric) peak is very similar to that of the $\mathrm{N} 1 \mathrm{~s}$ and therefore is not shown. We will present the gate-dependence of these peaks and discuss the underlying mechanism(s).

We started our investigation by measuring the electrical characteristics of the graphene transistor. Figure 2a shows the variation of the drain current (black curve) and the total resistance (red curve) of the graphene layer with the gate voltage. During the electrical measurements, we applied $1.0 \mathrm{~V}$ to the drain electrode while the source electrode was grounded. The resistivity of the device varied from 0.5 to $4 \mathrm{k} \Omega$ as we sweep the gate voltage from -40 to $50 \mathrm{~V}$. The transistor showed a p-type conduction with an on-off ratio of 8 , which is a typical number for back-gated transistors based on CVD graphene. ${ }^{46}$ The large charge neutral point at $60 \mathrm{~V}$ is due to the unintentional doping of graphene by the charged impurities on the substrate. Besides the gate-induced electrostatic doping, unintentional doping due to the substrate or transfer process and/or charge puddle formation are main factors that define the minimum charges on the graphene layer. The surface properties of the substrate (such as, surface chemistry, organic 
(a)

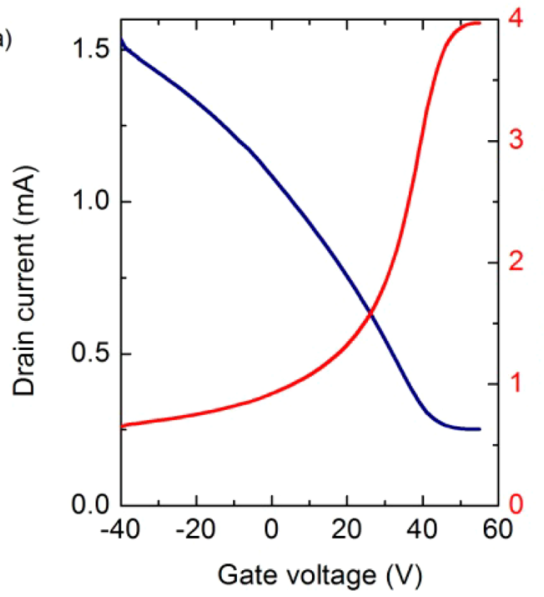

(b)

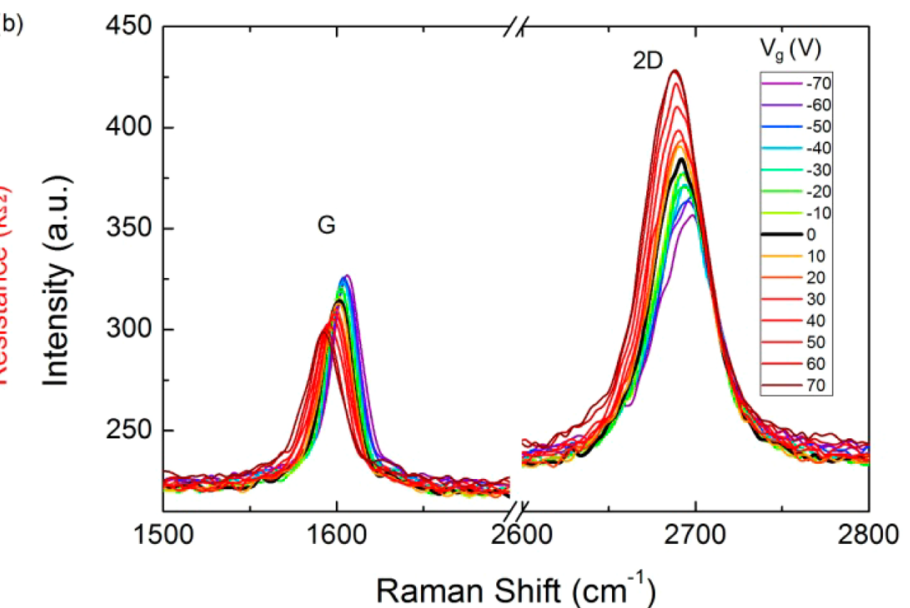

(c)

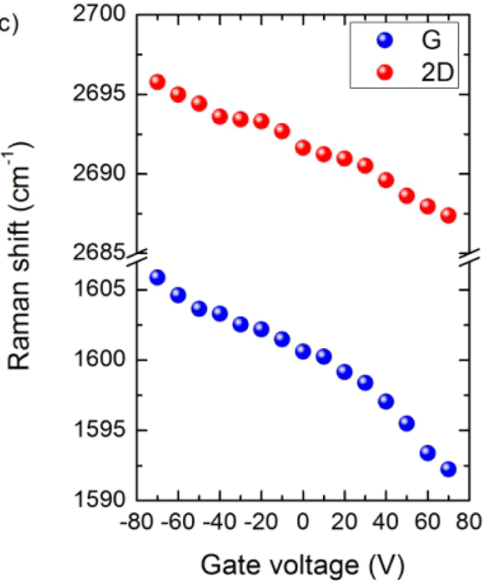

(d)

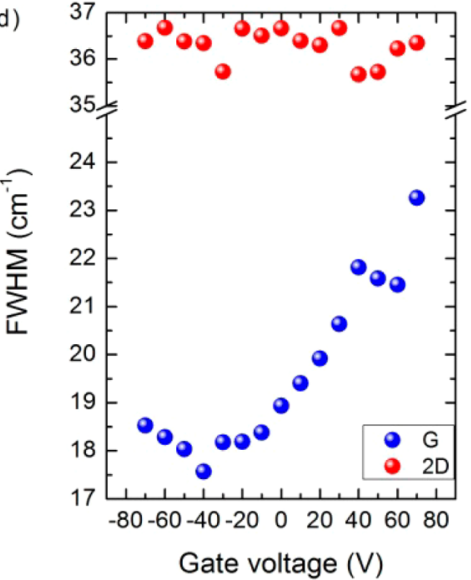

(e)

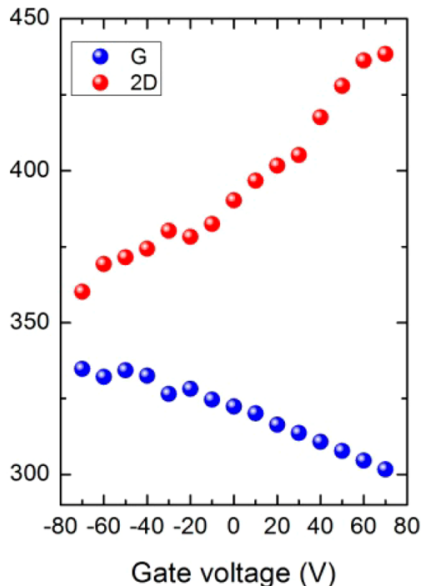

Figure 2. (a) Variation of the drain current with the gate voltage. The red curve shows the resistance of the graphene layer. The transistor shows a strong p-type behavior with a charge neutral point of $60 \mathrm{~V}$. (b) Raman spectra of graphene recorded at gate voltages between -70 to $70 \mathrm{~V}$. Both Gband and 2D-band shows profound variation with the gate voltage. The variation of Raman shift (c), fwhm (d), and Raman intensity (e) of G-band (blue dots) and 2D-band (red dots) of graphene.

residues, and the dielectric constant) determine the amount of induced charges on the graphene layer. This substrate induced unintentional doping appears in the Raman spectra as a shift in frequency and the intensity of the $\mathrm{G}$ and $2 \mathrm{D}$ bands. For example, on a $\mathrm{SiO}_{2}$ substrate graphene is p-type doped with Fermi energy of 150-200 meV.

After the electrical measurements, we recorded the Raman spectra of graphene (Figure $2 \mathrm{~b})$ at various gate voltages $(-70$ to $70 \mathrm{~V})$. The Raman spectra show profound gate-dependence and provide a useful piece of information about the electrostatic doping level and Fermi energy change of graphene. Graphene has two distinct Raman peaks called G-band (optical phonons at $\Gamma$ point) and $2 \mathrm{D}$ (overtone of the zone-boundary optical phonons). Fitting of G-band and 2D-band by Lorentzian functions yields the Raman frequency (Figure 2c), spectral width (Figure 2d, full width at half-maxima (fwhm)) and Raman intensity as a function of the gate voltage. The charge neutral point (so-called Dirac point) of graphene is at $60 \mathrm{~V}$. The gate-induced free carrier density on graphene can be written as $n_{\mathrm{g}}=C\left(V_{\mathrm{g}}-V_{\mathrm{CNP}}\right) / e$ where $C$ is the electrostatic capacitance of the gate dielectric, $V_{\mathrm{g}}$ is the gate voltage and $V_{\text {CNP }}$ is the charge neutral point and $e$ is the elementary charge. The modulation of carrier density significantly changes the frequency and lifetime of phonons. Figure $2 \mathrm{c}$ shows the extracted Raman frequency of $\mathrm{G}$ and $2 \mathrm{D}$ bands. The frequency change of G-band is particularly important for our study because it provides quantitative information for the Fermi energy. The electron-phonon coupling of massless Dirac electrons on graphene can be tuned by electrostatic doping. At the charge neutral point, the carrier concentration is at a minimum that is limited by the impurity-induced charge puddle formation on graphene. The Raman frequency of G-band at the charge neutral point $\left(\omega_{\mathrm{CNP}}^{\mathrm{G}}\right)$ is $1592 \mathrm{~cm}^{-1}$. The Raman frequency increases up to $1605 \mathrm{~cm}^{-1}$ as we sweep the gate voltage. The change in the Raman frequency is a linear function of Fermi energy that is $\Delta \omega^{\mathrm{G}}=\omega^{\mathrm{G}}-\omega_{\mathrm{CNP}}^{\mathrm{G}}=\lambda\left|E_{\mathrm{F}}\right|$, where $\lambda$ is the electron-phonon coupling strength. ${ }^{6}$ The maximum change in the Raman frequency is about $13 \mathrm{~cm}^{-1}$, corresponding to he Fermi energy of $300 \mathrm{meV}$. The corresponding charge density on graphene is around $5 \times 10^{12}$ $1 / \mathrm{cm}^{2}$. We also observed spectral narrowing of G-band indicating longer phonon lifetime due to blocking of possible damping mechanisms.

After obtaining information about the Fermi energy and electrostatic doping of graphene, we studied the gate voltage dependence of binding energy of $\mathrm{C} 1 \mathrm{~s}$ photoelectrons. The recorded XPS spectra of $\mathrm{C} 1 \mathrm{~s}$ region at various gate voltages are shown in Figure 3a. We observed a slight decrease in the binding energy with increasing charge density. The variation of the binding energies of $\mathrm{C} 1 \mathrm{~s}$ calculated by fitting of the spectra 

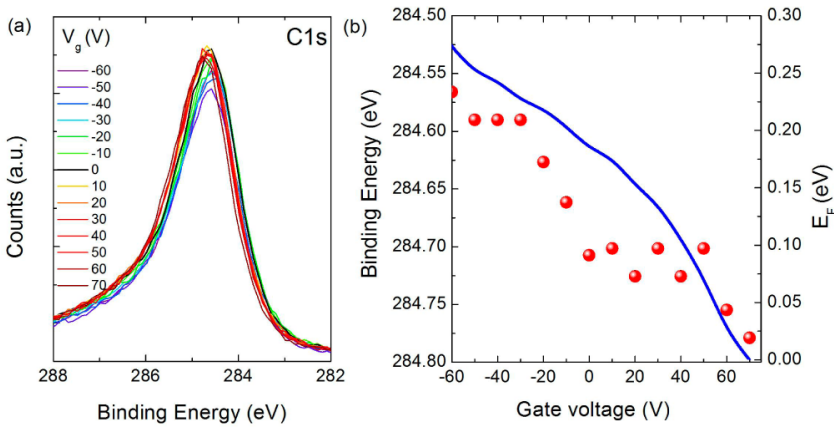

(c)

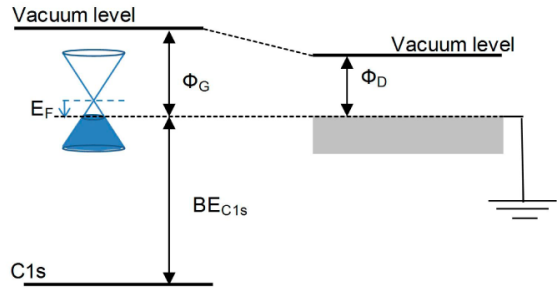

Graphene

Spectrometer

Figure 3. (a) C 1s region of the XPS spectrum of graphene transistor recorded at gate voltages between -60 to $70 \mathrm{~V}$. (b) The scattered points show the variation of the binding energy of the $\mathrm{C} 1 \mathrm{~s}$ as a function of gate voltage. The blue line shows the Fermi energy calculated from the shift of the Raman frequency of the G-band. (c) Schematic representation of the alignment of the band structure of graphene with respect to the Fermi energy of the spectrometer. During the XPS measurements, the graphene layer is grounded that aligns the Fermi energies of graphene and the spectrometer. The binding energy of $\mathrm{C} 1 \mathrm{~s}$ is measured with respect to the Fermi energy.

is given in Figure $3 \mathrm{~b}$ (scattered plot). The binding energy varies from 284.77 to $284.57 \mathrm{eV}$ for the gate voltage range. The solid line in Figure $3 \mathrm{~b}$ shows the Fermi energy calculated from the shift in the Raman frequency of the G-band. At $-60 \mathrm{~V}$, the Fermi energy is around $270 \mathrm{me}, \mathrm{V}$ which agrees well with the shift of the binding energy $200 \mathrm{meV}$. The shift of binding energy of $\mathrm{C}$ 1s can be understood from the alignment of the Fermi energies of graphene and the spectrometer. The schematic representation in Figure $3 c$ depicts the electronic band structure of graphene and the measured binding energy of C 1s $\left(\mathrm{BE}_{\mathrm{C} 1 \mathrm{~s}}\right) . \Phi_{\mathrm{G}}$ and $\Phi_{\mathrm{D}}$ represent the work function of graphene and the spectrometer, respectively. During the measurements, graphene was grounded to the spectrometer, which aligns the Fermi energies of graphene and the spectrometer. Because the binding energy of $C 1 \mathrm{~s}$ is measured with respect to the Fermi energy, the variation of Fermi energy with increasing charge density appears as a decrease in the binding energy.

Now we would like to analyze the gate dependence of the photoemission from the $\mathrm{Si}_{3} \mathrm{~N}_{4}$ gate dielectric underneath the graphene layer. In our previous work, we observed that presence of graphene on a dielectric substrate provides equipotential surfaces where the local potential of graphene alters the potential of the dielectric substrate. ${ }^{44}$ Therefore, binding energy of the photoelectrons emitted from the dielectric varies with the local voltage of graphene. Figure $4 \mathrm{a}$ depicts the cross-section of the graphene transistor. We measured N 1s and Si 2p and O 1s regions of the XPS spectra for various gate voltages while graphene is grounded. The changes in the $\mathrm{N} 1 \mathrm{~s}$ and $\mathrm{Si} 2 \mathrm{p}$ peaks with the gate voltages, mainly progressive shift and asymmetric broadening, are much more pronounced. In order to understand these variations one has to consider two effects simultaneously: (i) that the effective local voltage is increasing linearly, causing the progressive shift; and (ii) the signal is attenuated exponentially, causing the asymmetry, as one goes down into the depths of the gate dielectric. In this case, use of the Si $2 p$ peak, rather than the $\mathrm{N}$ $1 \mathrm{~s}$, is sounder because more experimental data exists for it. Accordingly, a simple model can be constructed by artificially dividing the top 10 layers of silicon nitride into $1 \mathrm{~nm}$ slices and using the Si $2 \mathrm{p}$ peak recorded when the gate was also grounded as the reference. Two operations are executed stepwise. (i) The reference peak is shifted by a certain amount $\left(\Delta E_{\text {inc }}\right)$, and (ii) its intensity is attenuated by the corresponding attenuation factor calculated using the formula $I_{\text {rec }}(x, E)=I_{0}(E) e^{-x / \alpha}$, where
$I_{0}$ is the intensity of the photoelectrons generated at the depth, $x, I_{0}(x, E)$ is the signal contributing from that depth after attenuation, and $\alpha$ is the attenuation length. The recorded spectra can be written as an integral of the photoelectron generated from different depth as

$$
I_{\text {rec }}(E)=\int_{0}^{t} I_{0}\left(E-\frac{V_{\mathrm{g}}}{t} x\right) e^{-x / \alpha} \mathrm{d} x
$$

where $t$ is the thickness of the gate dielectric and $V_{\mathrm{g}}$ is the gate voltage. The experimentally determined value of attenuation length for the $\mathrm{Si} 2 \mathrm{p}$ peak for $\mathrm{Si}_{3} \mathrm{~N}_{4}$ is $3.9 \mathrm{~nm} .{ }^{47}$ Implementation of this procedure and the resulting simulated spectra at only -20 and $+20 \mathrm{~V}$ gate voltages are shown in Figure $4 \mathrm{~d}$, together with the experimental spectra (scattered plot in Figure 4e). An excellent fit is obtained after shifting the spectrum by $0.2 \mathrm{eV} /$ $\mathrm{nm}$, matching perfectly the applied gate potential of $20 \mathrm{~V}$ over $100 \mathrm{~nm}$ gate length. Note also that extent of the gate-tunable shifts of the $\mathrm{O} 1 \mathrm{~s}$ is larger than that of $\mathrm{C} 1 \mathrm{~s}$ but much smaller than those of $\mathrm{N} 1 \mathrm{~s}$ and $\mathrm{Si} 2 \mathrm{p}$. This simple observation leads us to assign it predominantly to the surface oxide of the dielectric layer rather than any potential oxidized carbon moieties of the graphene layer.

These findings extracted from the unique experimental methodology implemented by simple modification of the 6 decades-old XPS technique has far more reaching consequences when utilized for assessing material and/or device properties. For example, local voltage variations due to chemical and/or physical defects, or on specific groups like $\mathrm{C} 1 \mathrm{~s}$ or $\mathrm{O} 1 \mathrm{~s}$ of oxidized samples ( $-\mathrm{CO}-$ or $-\mathrm{COO}-$, and so forth) as a result of chemical doping, or $\mathrm{N} \mathrm{1s}$ of the unwanted but unavoidable oxidized surface moieties, are expected to be significantly different when compared to pristine samples/devices. Other deleterious behaviors such as shorting paths, dielectric breakdown, and so forth are also within the reach. Moreover, the quality or the performance of the dielectric layer is also assessable, which can reveal vital information about the interface between the dielectric layer and the gate, hence the performance of the device. Another simple variation of the methodology can be employed to investigate the dynamics of electrical potential developments by imposing time-varying electrical stimuli, like square-wave-modulation, as was also shown in our previous work. ${ }^{43}$ Such measurements are in our agenda, and we advocate that they will impact greatly on furthering of our understanding of various materials and devices. 

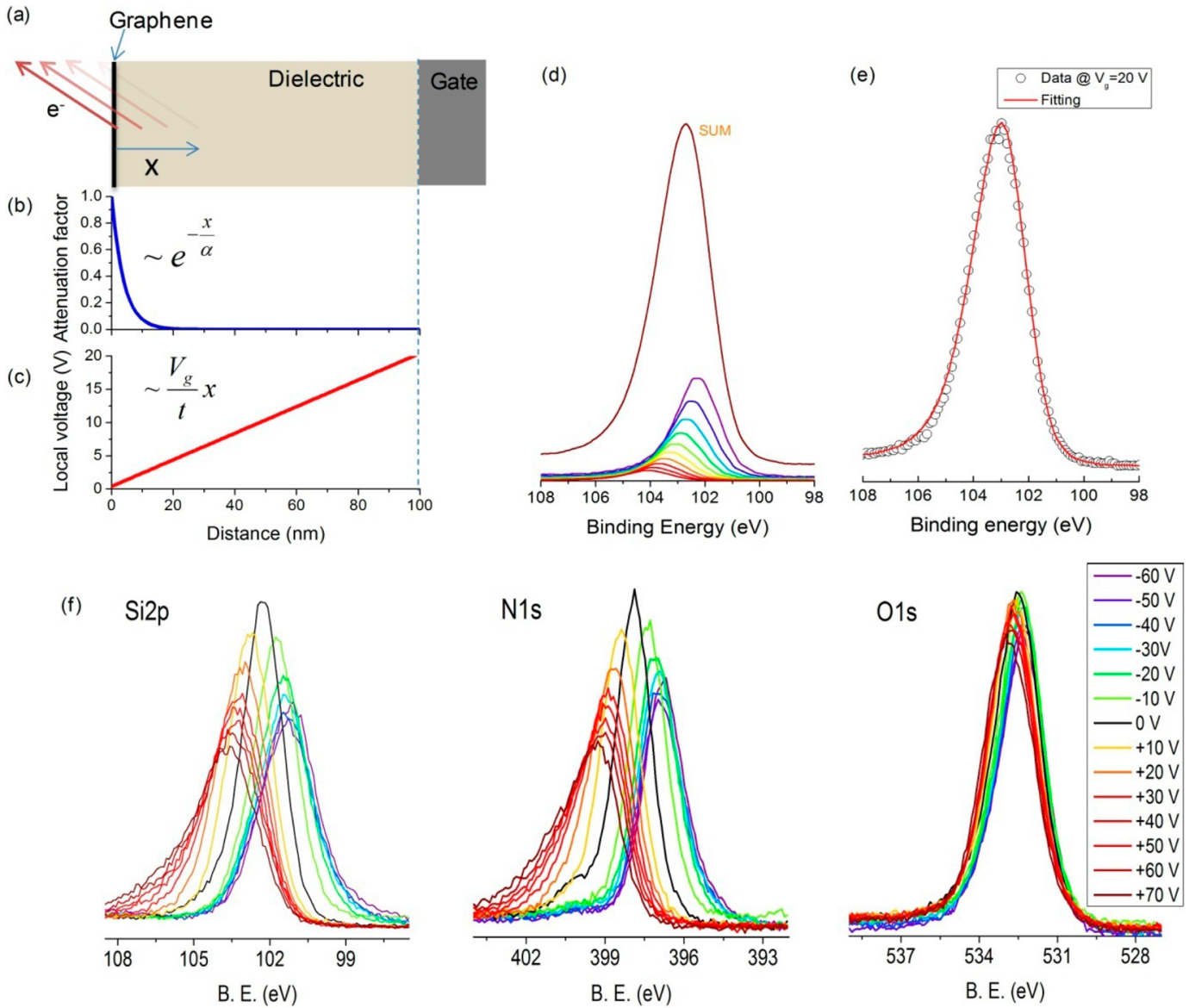

Figure 4. (a) Cross-sectional view of the back-gated graphene transistor. (b) Exponential attenuation of the intensity of the photoelectrons throughout the depth of the gate dielectric. (c) Variation of the local voltage as a function of position due to the electric field of the gate electrode biased at $20 \mathrm{~V}$. (d) Simulated Si $2 \mathrm{p}$ spectra obtained by shifting the peak position by the local voltage and multiplying by the attenuation factor. The sum represents the simulated XPS spectrum. (e) The measured and simulated XPS spectra for a gate bias of 20 V. (f) Si 2 p, N 1s, and O 1s regions of the XPS spectrum of graphene transistor recorded at gate bias between -60 to $70 \mathrm{~V}$.

Gate-tunable XPS of graphene transistors yields various chemically specific insights about the operation of the graphene transistors. Gate-induced shift of the Fermi energy and the gateinduced local voltage in the gate dielectric are the two main physical mechanisms behind observed shift in the binding energies. (1) Tracing the binding energy of $C 1 s$ that originates from the graphene layer provides the local Fermi energy. C 1s photoelectrons originate from other sources such as organic residues on graphene or chemically modified graphene can be identified from XPS spectra. The gate dependence of XPS could provide the local charge density and Fermi energy and associated local chemical information. This information is especially important for chemically synthesized graphene that has various forms of functional groups and defects. (2) Gateinduced local voltage provides unprecedented ability to identify the chemical content and its location in the dielectric layer. For example, in our experiments, the gate dependence of $\mathrm{O} 1 \mathrm{~s}$ suggests that the location of oxygen is close to the graphenedielectric interface. Ability to identify the chemical content and Local Fermi energy could be used for elucidating local Fermi energy, interface traps for transistors based on 2D layered materials, and heterojunctions. Internal photoemission spectroscopy (IPE) has been also used to measure the band alignment and work function of p-type doped graphene on $\mathrm{SiO}_{2}$ substrate. ${ }^{48}$ Because this method is based on transport measurements, the chemical information about the photo- electrons is lost. The gate-tunable photoemission and internal photoemission spectroscopy could be complementary techniques to elucidate the operation of transistors based on twodimensional materials.

In summary, we studied the gate-tunable photoemission spectra from a graphene transistor. This back-gated graphene transistor provides a tunable $2 \mathrm{D}$-electronic system that can be probed by electron-based surface characterization tools. Because XPS provides chemical specific information linked with the local electrical potential, we were able to determine the underlying physical mechanisms of the gate-voltage dependence of the binding energy of emitted photoelectrons. We observed that the shift of Fermi energy of graphene alters the measured binding energy of $\mathrm{C} 1 \mathrm{~s}$ electrons emitted from the graphene layer whereas the local potential on the gate dielectric yields progressive shift and asymmetric broadening of the $\mathrm{N} 1 \mathrm{~s}$ and $\mathrm{Si} 2 \mathrm{p}$ peaks originated from the dielectric material. Furthermore, we observed that $\mathrm{O}$ 1s electron originated from the surface of the dielectric behaves in a different way than $\mathrm{N}$ 1s. The gradual change of the local potential in the gate dielectric induces an asymmetry of the XPS signal and provides depth dependent information. We anticipate that the simultaneous chemical and electrical characterization with XPS will provide unique advantages to elucidate the operation and performance of electronic devices based on layered materials. We also expect that our methodology will be 
adopted by many researchers in the field as its implementation is quite simple.

\section{AUTHOR INFORMATION}

\section{Corresponding Authors}

*E-mail: (C.K.) ckocabas@fen.bilkent.edu.tr.

*E-mail: (S.S.) suzer@fen.bilkent.edu.tr.

\section{Notes}

The authors declare no competing financial interest.

\section{ACKNOWLEDGMENTS}

This work was supported by the Scientific and Technological Research Council of Turkey (TUBITAK) Grants 112T686, 113F278, and 212M051, and Marie Curie International Reintegration Grant (IRG) 256458.

\section{REFERENCES}

(1) Wang, F.; Zhang, Y. B.; Tian, C. S.; Girit, C.; Zettl, A.; Crommie, M.; Shen, Y. R. Science 2008, 320 (5873), 206-209.

(2) Novoselov, K. S.; Geim, A. K.; Morozov, S. V.; Jiang, D.; Katsnelson, M. I.; Grigorieva, I. V.; Dubonos, S. V.; Firsov, A. A. Nature 2005, 438 (7065), 197-200.

(3) Li, Z. Q.; Henriksen, E. A.; Jiang, Z.; Hao, Z.; Martin, M. C.; Kim, P.; Stormer, H. L.; Basov, D. N. Nature Phys. 2008, 4 (7), 532-535.

(4) Ando, T.; Fowler, A. B.; Stern, F. Rev. Mod. Phys. 1982, 54 (2), 437-672.

(5) Koppens, F. H. L.; Chang, D. E.; de Abajo, F. J. G. Nano Lett. 2011, 11 (8), 3370-3377.

(6) Yan, J.; Zhang, Y. B.; Kim, P.; Pinczuk, A. Phys. Rev. Lett. 2007, 98 (16), 166802 (4).

(7) Mak, K. F.; Ju, L.; Wang, F.; Heinz, T. F. Solid State Commun. 2012, 152 (15), 1341-1349.

(8) Polat, E. O.; Kocabas, C. Nano Lett. 2013, 13 (12), 5851-5857.

(9) Zhang, X.; Liu, M.; Yin, X. B.; Ulin-Avila, E.; Geng, B. S.; Zentgraf, T.; Ju, L.; Wang, F. Nature 2011, 474 (7349), 64-67.

(10) Liu, M.; Yin, X. B.; Zhang, X. Nano Lett. 2012, 12 (3), 14821485.

(11) Horng, J.; Chen, C. F.; Geng, B. S.; Girit, C.; Zhang, Y. B.; Hao, Z.; Bechtel, H. A.; Martin, M.; Zettl, A.; Crommie, M. F.; Shen, Y. R.; Wang, F. Phys. Rev. B 2011, 83 (16), 165113(5).

(12) Jablan, M.; Buljan, H.; Soljacic, M. Phys. Rev. B 2009, 80 (24), 245435(7).

(13) Grigorenko, A. N.; Polini, M.; Novoselov, K. S. Nat. Photonics 2012, 6 (11), 749-758.

(14) Ju, L.; Geng, B. S.; Horng, J.; Girit, C.; Martin, M.; Hao, Z.; Bechtel, H. A.; Liang, X. G.; Zettl, A.; Shen, Y. R.; Wang, F. Nat. Nanotechnol. 2011, 6 (10), 630-634.

(15) Fang, Z.; Wang, Y.; Schlather, A. E.; Liu, Z.; Ajayan, P. M.; García de Abajo, F. J.; Nordlander, P.; Zhu, X.; Halas, N. J. Nano Lett. 2014, 14 (1), 299-304.

(16) Ferrari, A. C. Solid State Commun. 2007, 143 (1-2), 47-57.

(17) Das, A.; Pisana, S.; Chakraborty, B.; Piscanec, S.; Saha, S. K.; Waghmare, U. V.; Novoselov, K. S.; Krishnamurthy, H. R.; Geim, A. K.; Ferrari, A. C.; Sood, A. K. Nat. Nanotechnol. 2008, 3 (4), 210-215.

(18) Wang, F.; Chen, C. F.; Park, C. H.; Boudouris, B. W.; Horng, J.; Geng, B. S.; Girit, C.; Zettl, A.; Crommie, M. F.; Segalman, R. A.; Louie, S. G. Nature 2011, 471 (7340), 617-620.

(19) Yan, L.; Punckt, C.; Aksay, I. A.; Mertin, W.; Bacher, G. Nano Lett. 2011, 11 (9), 3543-3549.

(20) Yu, Y. J.; Zhao, Y.; Ryu, S.; Brus, L. E.; Kim, K. S.; Kim, P. Nano Lett. 2009, 9 (10), 3430-3434.

(21) Bostwick, A.; Ohta, T.; Horn, K.; Rottenberg, A. Nat. Phys. 2007, 3, 36-40.

(22) Wintterlin, J.; Bocquet, M.-L. Surf. Sci. 2009, 603, 1841-1852.

(23) Batzill, M. Surf. Sci. Rep. 2012, 67, 83-115.
(24) Wei, P.; Liu, N.; Lee, H. R.; Adijanto, E.; Ci, L. J.; Naab, B. D.; Zhong, J. Q.; Park, J.; Chen, W.; Cui, Y.; Bao, Z. A. Nano Lett. 2013, 13 (5), 1890-1897.

(25) Heo, J.; Byun, K.-E.; Lee, J.; Chung, H.-J.; Jeon, S.; Park, S.; Hwang, S. Nano Lett. 2013, 13 (12), 5967-5971.

(26) Lussem, B.; Tietze, M. L.; Kleemann, H.; Hossbach, C.; Bartha, J. W.; Zakhidov, A.; Leo, K. Nat. Commun. 2013, 4, 2775 (6).

(27) Yang, D.; Velamakanni, A.; Bozoklu, G.; Park, S.; Stoller, M.; Piner, R. D.; Stankovich, S.; Jung, I.; Field, D. A.; Ventrice, C. A.; Ruoff, R. S. Carbon 2009, 47 (1), 145-152.

(28) Brako, R.; Sokcevic, D.; Lazic, P.; Atodiresei, N. New J. Phys. 2010, 12, 113016(17).

(29) Busse, C.; Lazic, P.; Djemour, R.; Coraux, J.; Gerber, T.; Atodiresei, N.; Caciuc, V.; Brako, R.; N’Diaye, A. T.; Blugel, S.; Zegenhagen, J.; Michely, T. Phys. Rev. Lett. 2011, 107 (3), 036101 (4).

(30) Chiu, P. L.; Mastrogiovanni, D. D. T.; Wei, D. G.; Louis, C.; Jeong, M.; Yu, G.; Saad, P.; Flach, C. R.; Mendelsohn, R.; Garfunkel, E.; He, H. X. J. Am. Chem. Soc. 2012, 134 (13), 5850-5856.

(31) Eckmann, A.; Felten, A.; Mishchenko, A.; Britnell, L.; Krupke, R; Novoselov, K. S.; Casiraghi, C. Nano Lett. 2012, 12 (8), 39253930.

(32) Filleter, T.; Emtsev, K. V.; Seyller, T.; Bennewitz, R. Appl. Phys. Lett. 2008, 93 (13), 133117(3).

(33) Hammock, M. L.; Sokolov, A. N.; Stoltenberg, R. M.; Naab, B. D.; Bao, Z. A. ACS Nano 2012, 6 (4), 3100-3108.

(34) Kim, M.; Safron, N. S.; Huang, C. H.; Arnold, M. S.; Gopalan, P. Nano Lett. 2012, 12 (1), 182-187.

(35) Larciprete, R.; Lacovig, P.; Gardonio, S.; Baraldi, A.; Lizzit, S. J. Phys. Chem. C 2012, 116 (18), 9900-9908.

(36) Perera, S. D.; Mariano, R. G.; Vu, K.; Nour, N.; Seitz, O.; Chabal, Y.; Balkus, K. J. ACS Catal. 2012, 2 (6), 949-956.

(37) Prezioso, S.; Perrozzi, M.; Donarelli, M.; Bisti, F.; Santucci, S.; Palladino, L.; Nardone, M.; Treossi, E.; Palermo, V.; Ottaviano, L. Langmuir 2012, 28 (12), 5489-5495.

(38) Rana, K.; Kucukayan-Dogu, G.; Sen, H. S.; Boothroyd, C.; Gulseren, O.; Bengu, E. J. Phys. Chem. C 2012, 116 (20), 1136411369.

(39) Usachov, D.; Vilkov, O.; Gruneis, A.; Haberer, D.; Fedorov, A.; Adamchuk, V. K.; Preobrajenski, A. B.; Dudin, P.; Barinov, A.; Oehzelt, M.; Laubschat, C.; Vyalikh, D. V. Nano Lett. 2011, 11 (12), 54015407.

(40) Wang, S. N.; Wang, R.; Liu, X. F.; Wang, X. W.; Zhang, D. D.; Guo, Y. J.; Qiu, X. H. J. Phys. Chem. C 2012, 116 (19), 10702-10707.

(41) Wei, D. C.; Liu, Y. Q.; Wang, Y.; Zhang, H. L.; Huang, L. P.; Yu, G. Nano Lett. 2009, 9 (5), 1752-1758.

(42) Tarabek, J.; Kavan, L.; Dunsch, L.; Kallbac, M. J. Phys. Chem. C 2008, 112 (36), 13856-13861.

(43) Sezen, H.; Suzer, S. Thin Solid Films 2013, 534 (1), 1-11.

(44) Kocabas, C.; Suzer, S. Anal. Chem. 2013, 85 (8), 4172-4177.

(45) Salihoglu, O.; Balci, S.; Kocabas, C. Appl. Phys. Lett. 2012, 100 (21), 213110 (5).

(46) Balci, O.; Kocabas, C. Appl. Phys. Lett. 2012, 101 (24), 243105 (5).

(47) Jablonski, A.; Powell, C. J. Surf. Sci. 2002, 520 (1-2), 78-96. (48) Xu, K.; Zeng, C.; Zhang, Q.; Yan, R.; Ye, P.; Wang, K.; Seabaugh, A. C.; Xing, H. G.; Suehle, J. S.; Richter, K. A.; Gundlach, D. J.; Nguyen, N. V. Nano Lett. 2013, 13 (1), 131-136. 